\title{
Protective effects of $\alpha$-2-macroglobulin on human bone marrow mesenchymal stem cells in radiation injury
}

\author{
YANG LIU $^{1 *}$, WANTING CAO ${ }^{1 *}$, XIANGBO KONG $^{2}$, JIE LI $^{1}$, XUEYING CHEN ${ }^{1}$, \\ YAPING GE ${ }^{1}$, WANZHEN ZHONG ${ }^{1}$ and SILIAN FANG ${ }^{1}$ \\ ${ }^{1}$ Department of Oral and Maxillofacial Surgery, The Sixth Affiliated Hospital of Sun Yat-Sen University, \\ Guangzhou, Guangdong 510655; ${ }^{2}$ Department of Stomatology, Sun Yat-Sen Memorial Hospital of \\ Sun Yat-Sen University, Guangzhou, Guangdong 510120, P.R. China
}

Received January 16, 2018; Accepted July 20, 2018

DOI: $10.3892 / \mathrm{mmr} .2018 .9449$

\begin{abstract}
Osteoradionecrosis of the jaws (ORNJ) is a complication of oral and maxillofacial malignancy that arises following radiotherapy; progressive jaw necrosis severely decreases the quality of life of patients. Human bone marrow mesenchymal stem cells (hBMMSCs) are a cell type with self-renewal and pluripotent differentiation potential in the bone marrow stroma. These cells are associated with bone tissue regeneration and are one of the primary cell types affected by bone tissue radiation injury. $\alpha-2$-macroglobulin $\left(\alpha_{2} \mathrm{M}\right)$ is a glycoprotein-rich macromolecule that interacts with cytokines, growth factors and hormones to serve a variety of biological roles. In addition, $\alpha_{2} \mathrm{M}$ possesses radio-protective effects. The aim of the present study was to investigate whether $\alpha_{2} \mathrm{M}$ has protective effects against radiation injury of hBMMSCs. Cell counting kit- 8 and colony formation assays were used to monitor cell proliferation. Western blot analysis and reverse transcription-quantitative polymerase chain reaction were used to detect Beclin1, microtubule-associated protein $1 \mathrm{~A} / 1 \mathrm{~B}$, sex determining region $\mathrm{Y}$, Nanog, runt-related transcription factor 2, osteoglycin and manganese superoxide dismutase expression. The formation of calcium nodules was evaluated by Alizarin red staining after osteogenic induction. Flow cytometric analysis of Annexin-V and propidium iodide double staining was used to detect changes in apoptosis rate. Alkaline phosphatase and superoxide dismutase activity were determined using colorimetric assays. Reactive oxygen species levels were detected using 2',7'-dichlorodihydrofluorescein
\end{abstract}

Correspondence to: Dr Silian Fang, Department of Oral and Maxillofacial Surgery, The Sixth Affiliated Hospital of Sun Yat-Sen University, 26 Yuancun Er Heng Road, Guangzhou, Guangdong 510655, P.R. China

E-mail: fangsilian@126.com

*Contributed equally

Key words: radiation injury, $\alpha$-2-macroglobulin, human bone marrow mesenchymal stem cells, protective effects diacetate. The results of the present study revealed that $\alpha_{2} \mathrm{M}$ increased the rate of proliferation, reduced autophagy, alleviated pluripotent differentiation injury, increased the osteogenic differentiation ability and decreased the rate of apoptosis in hBMMSCs following irradiation via an antioxidative pathway. In conclusion, $\alpha_{2} \mathrm{M}$ exhibited protective effects against radiation injury in hBMMSCs and may be considered a potential therapeutic agent for the prevention and treatment of ORNJ.

\section{Introduction}

At present, radiation therapy is one of the primary means of treatment for malignant tumors. When radiation is used to eliminate tumor cells, the adjacent normal tissues and organs are unavoidably exposed, causing various complications (1). Osteoradionecrosis of the jaws (ORNJ) is a serious complication of oral and maxillofacial malignancies that commonly follows a regimen of radiotherapy treatment. Symptoms in patients include tissue necrosis, exposure of dead bone and pathological fractures $(2,3)$.

Human bone marrow mesenchymal stem cells (hBMMSCs) are cells with pluripotent differentiation potential, which can differentiate into osteoblasts, adipocytes and chondrocytes. hBMMSCs are also one of the major target cell types involved in damage of bone tissue via radiation $(4,5)$. It has been demonstrated that radiation can induce hBMMSC proliferative abnormalities, genomic DNA damage and micronucleus formation (6); radiation has been reported to inhibit osteogenic differentiation in hBMMSCs (6).

Previously, the authors reported that $\alpha$-2-macroglobulin $\left(\alpha_{2} \mathrm{M}\right)$ is expressed at low levels in the serum of patients with ORNJ, which was determined via the analysis of clinical samples $(7,8)$. In addition, ELISA demonstrated a positive correlation between $\alpha_{2} \mathrm{M}$ expression and radiotherapy dosage in patients who received radiotherapy for the treatment of nasopharyngeal carcinoma (9). This suggested that $\alpha_{2} \mathrm{M}$ is closely associated with radiation injury in the jaw bone; however, the underlying mechanism is unknown. $\alpha_{2} \mathrm{M}$ is an important macromolecular glycoprotein in the plasma and belongs to a family of broad-spectrum protease inhibitors (10). Accumulating data has demonstrated that $\alpha_{2}$ M possesses a wide range of biological functions, including radioprotective, 
anti-infective and anti-inflammatory effects (11). In addition, $\alpha_{2} \mathrm{M}$ was reported to exhibit protection against damage from anti-ionizing radiation (12).

In the present study, the protective effects of $\alpha_{2} \mathrm{M}$ on hBMMSCs exposed to radiation injury were investigated by applying various concentrations of $\alpha_{2} \mathrm{M}$ to the cell medium following exposure to ionizing radiation.

\section{Materials and methods}

Materials and reagents. $\alpha_{2} \mathrm{M}$ was obtained from Enzo Life Sciences (Farmingdale, NY, USA). hBMMSCs were obtained from Cyagen Biosciences (Guangzhou) Inc. (HUXMA-01001; Guangzhou, China). Dulbecco's modified Eagle's medium (DMEM), fetal bovine serum (FBS), penicillin-streptomycin mixture and $0.25 \%$ trypsin-EDTA were purchased from Gibco (Thermo Fisher Scientific, Inc., Waltham, MA, USA). Osteogenic induction medium was purchased from Cyagen Biosciences (Guangzhou) Inc. (GUXMX-90021). Antibodies against Beclin1 (1:1,000; cat. no. 11306-1-AP), microtubule-associated protein 1A/1B (LC-3; 1:1,000; cat. no. 14600-1-AP), sex determining region Y (Sox 2; 1:5,000; cat. no. 11064-1-AP), Nanog (1:2,000; cat. no. 14295-1-AP), manganese superoxide dismutase (MnSOD; 1:3,000; cat. no. 24127-1-AP) and GAPDH (1:10,000; cat. no. 60004-1-Ig) were purchased from ProteinTech Group (Chicago, IL, USA). The runt-related transcription factor 2 (RUNX2; 1:1,000; cat. no. 12556) antibody was purchased from Cell Signaling Technology, Inc. (Danvers, MA, USA), the osteoglycin (OGN; 1:1,000; cat. no. sc-365228) antibody was obtained from Santa Cruz Biotechnology, Inc. (Dallas, TX, USA), and the goat anti-rabbit (1:10,000; cat. no. ab6721) and goat anti-mouse (1:10,000; cat. no. ab6789) horseradish peroxidase (HRP) conjugated secondary antibodies were obtained from Abcam (Cambridge, MA, USA).

Culture of hBMMSCs. hBMMSCs were cultured in DMEM supplemented with $10 \%$ FBS and $1 \%$ penicillin-streptomycin at $37^{\circ} \mathrm{C}$ and $5 \% \mathrm{CO}_{2}$ with saturated humidity. The culture medium was replaced every 3 days. When the cells reached $80 \%$ confluence, they were digested with trypsin and passaged at a 1:3 ratio. The fourth generation of cells was used for subsequent analysis.

$\alpha_{2} M$ solution preparation and cell irradiation. A total of $1 \mathrm{mg}$ $\alpha_{2} \mathrm{M}$ was dissolved in $0.5 \mathrm{ml}$ sterilized $\mathrm{ddH}_{2} \mathrm{O}$ to prepare a solution of $2.0 \mathrm{mg} / \mathrm{ml} \alpha_{2} \mathrm{M}$, which was stored at $-20^{\circ} \mathrm{C}$. X-ray irradiation was performed when hBMMSCs attained $70 \%$ confluence. hBMMSCs were irradiated at the Sun Yat-Sen University Medical Experimental Center (Guangzhou, China). An RS2000 X-ray irradiator (Radsource, LLC, Brentwood, TN, USA) was used at a voltage of $160 \mathrm{kV}$, a current of $25 \mathrm{~mA}$, and a dose rate of $1.24 \mathrm{~Gy} / \mathrm{min}$. Cells were irradiated at room temperature for $1.61,3.23,6.45$ and $9.68 \mathrm{~min}$ to obtain a dose of 2, 4, 8 and $12 \mathrm{~Gy}$, respectively.

Experimental groups. hBMMSCs were randomly divided into the following groups: Blank control, radiation control, 0.25 and $0.50 \mathrm{mg} / \mathrm{ml} \alpha_{2} \mathrm{M}$, respectively. The blank control group was not administered any treatment, whereas the remaining three groups received X-ray irradiation. The $\alpha_{2} \mathrm{M}$ treatment groups were supplemented with $\alpha_{2} \mathrm{M}$ to the final concentrations of 0.25 and $0.50 \mathrm{mg} / \mathrm{ml}$, respectively, in the complete medium (DMEM supplemented with $10 \%$ FBS and $1 \%$ penicillin-streptomycin) and osteogenic induction medium (DMEM supplemented with $10 \% \mathrm{FBS}, 1 \%$ penicillin-streptomycin, $50 \mathrm{mg} / 1$ ascorbic acid, $10 \mathrm{mmol} / \mathrm{l} \beta$-glycerophosphate and $1 \mu \mathrm{mol} / \mathrm{l}$ dexamethasone). Following irradiation, cells in complete medium were treated with $\alpha 2 \mathrm{M}$ for $24 \mathrm{~h}$, whereas cells in the osteogenic induction medium were treated with $\alpha 2 \mathrm{M}$ for 3 days at $37^{\circ} \mathrm{C}$.

Cell Counting Kit-8 (CCK-8) assay. hBMMSCs were seeded into 96 -well plates at a density of $5 \times 10^{3}$ cells/well, with five wells per group. Following adhesion to the well, the cells were exposed to various doses of $\mathrm{X}$-ray radiation, to explore the relationship between dose and cell proliferation. After incubation for 1, 3, 5 and 7 days, $10 \mu$ CCK-8 (Dojindo Molecular Technologies, Inc., Kumamoto, Japan) solution was added to each well. Following incubation for $2 \mathrm{~h}$, the absorbance was measured at a wavelength of $450 \mathrm{~nm}$ using a 96-well-plate reader (Thermo Fisher Scientific, Inc.).

Colony formation assay. hBMMSCs were seeded at 100 cells/well and cultured in 6-cm petri dishes. After 2 weeks culture in complete medium at $37^{\circ} \mathrm{C}$ and $5 \% \mathrm{CO}_{2}$ with saturated humidity, the cells were washed twice with PBS, fixed with $4 \%$ paraformaldehyde for $20 \mathrm{~min}$ at room temperature, washed twice with ddH2O, and stained with $0.1 \%$ crystal violet (Sigma-Aldrich; Merck KGaA, Darmstadt, Germany) for $15 \mathrm{~min}$ at room temperature. Images of the colonies were obtained using an Epson scanner (Seiko Epson Corp., Suwa, Japan). Then, images were collected for analysis.

Reverse transcription-quantitative polymerase chain reaction (RT-qPCR) analysis. hBMMSCs were seeded into 6-well plates at a density of $4 \times 10^{5}$ cells/well, and total RNA was extracted using TRIzol ${ }^{\circledR}$ reagent (Invitrogen; Thermo Fisher Scientific, Inc.). RNA was reverse transcribed into cDNA using a PrimeScript ${ }^{\mathrm{TM}}$ RT Reagent Kit with gDNA Eraser (Takara Bio, Inc., Otsu, Japan), according to the manufacturer's instructions and amplified using a SYBR ${ }^{\circledR}$ Premix Ex Taq ${ }^{\mathrm{TM}}$ II kit (Takara Bio, Inc.). The PCR cycling conditions were as follows: $95^{\circ} \mathrm{C}$ for $30 \mathrm{sec}$, followed by 40 cycles of $95^{\circ} \mathrm{C}$ for $5 \mathrm{sec}$ and $60^{\circ} \mathrm{C}$ for $20 \mathrm{sec}$. The housekeeping gene GAPDH was used for normalization, and the relative expression was calculated by the $2^{-\Delta \Delta \mathrm{Cq}}$ relative quantitation method (13). The primers used are listed in Table I. All primer sequences were determined via established GenBank sequences (https://www.ncbi.nlm.nih.gov/genbank/).

Western blot analysis. hBMMSCs were seeded into 6-well plates at a density of $4 \times 10^{5}$ cells/well, and total protein was extracted using radioimmunoprecipitation assay (RIPA) lysis buffer (Beyotime Institute of Biotechnology, Haimen, China). Protein concentration was determined via a Bicinchoninic Acid (BCA) kit (Thermo Fisher Scientific, Inc.). A total of $30 \mu \mathrm{g}$ protein was separated by $10 \%$ SDS-PAGE (Beyotime Institute of Biotechnology) at $120 \mathrm{~V}$ for $\sim 1.5 \mathrm{~h}$. Following the completion of electrophoresis, proteins were transferred to a polyvinylidiene difluoride membrane at a current of $280 \mathrm{~mA}$ for $\sim 90 \mathrm{~min}$. The membrane was blocked in 5\% skim milk for $1 \mathrm{~h}$ at room 
Table I. Primer sequences.

\begin{tabular}{|c|c|c|}
\hline Gene & Primer sequences $\left(5^{\prime}-3^{\prime}\right)$ & NCBI reference sequences \\
\hline \multicolumn{3}{|c|}{ Beclin 1} \\
\hline $\mathrm{F}$ & ATGCAGGTGAGCTTCGTGTG & NC_000017.11 \\
\hline $\mathrm{R}$ & CTGGGCTGTGGTAAGTAATGGA & \\
\hline \multicolumn{3}{|l|}{$\mathrm{LC}-3$} \\
\hline $\mathrm{F}$ & AGTTGGCACAAACGCAGGGTA & NC_000020.11 \\
\hline $\mathrm{R}$ & TTAGGAGTCAGGGACCTTCAGCA & \\
\hline \multicolumn{3}{|l|}{ Sox 2} \\
\hline $\mathrm{F}$ & GTGAGCGCCCTGCAGTACAA & NC_000003.12 \\
\hline $\mathrm{R}$ & GCGAGTAGGACATGCTGTAGGTG & \\
\hline \multicolumn{3}{|c|}{ Nanog } \\
\hline $\mathrm{F}$ & САTTCTCCTGCCTCAGCCTC & NC_000012.12 \\
\hline $\mathrm{R}$ & CCCGTCTCTACTAAAAATACAAAA & \\
\hline \multicolumn{3}{|c|}{ RUNX2 } \\
\hline $\mathrm{F}$ & CTTTGTAGCACAAACATTGCTGGA & NC_000006.12 \\
\hline $\mathrm{R}$ & CAAAGCTGTGGTACCTGTTCTGGA & \\
\hline \multicolumn{3}{|l|}{ OGN } \\
\hline $\mathrm{F}$ & GGTGCCAGTGCACACTACCTTC & NC_000009.12 \\
\hline $\mathrm{R}$ & CTCTGGCACCACTGAGTCATCATAA & \\
\hline \multicolumn{3}{|c|}{ GAPDH } \\
\hline $\mathrm{F}$ & AGGCTAGCTGGCCCGATTTC & NC_000012.12 \\
\hline $\mathrm{R}$ & TGGCAACAATATCCACTTTACCAGA & \\
\hline
\end{tabular}

temperature and then incubated at $4^{\circ} \mathrm{C}$ overnight with primary antibodies. The membrane was washed three times in tris-buffered saline with $0.1 \%$ Tween-20 (TBST) three times, followed by incubation with secondary antibodies at room temperature for $1 \mathrm{~h}$. The membrane was washed three times with TBST, then treated with an Immobilon Western Chemiluminescent HRP Substrate (EMD Millipore, Billerica, MA, USA), according to the manufacturer's protocols. The grayscale values of the bands were analyzed by using ImageJ software v. 1.47 (National Institutes of Health, Bethesda, MA, USA) and the expression levels of the target protein were calculated by normalizing the gray value of the target protein to that of GAPDH.

Osteogenic differentiation. When hBMMSCs attained $70 \%$ confluence, the complete medium was replaced with osteogenic induction differentiation medium, and cells were cultured at $37^{\circ} \mathrm{C}$ and $5 \% \mathrm{CO}_{2}$ with saturated humidity. The induction medium was replaced every 3 days. The gene expression of RUNX2 and OGN was detected following 1 and 2 weeks of culture in osteogenesis induction medium, respectively.

Alkaline phosphatase (ALP) activity assay. hBMMSCs were seeded into 12 -well plates at a density of $4 \times 10^{4}$ cells/well, and $200 \mu$ l of $1 \%$ Triton X-100 (Beyotime Institute of Biotechnology) was added to each well for lysis overnight at $4^{\circ} \mathrm{C}$. Following the completion of lysis, $30 \mu 1$ lysate was obtained for analysis. An ALP assay kit (Nanjing Jiancheng Bioengineering Institute, Nanjing, China) was used according to the manufacturer's protocols to determine the absorbance at a wavelength of $520 \mathrm{~nm}$, using a 96-well-plate reader (Thermo Fisher Scientific, Inc.). An additional $10 \mu 1$ lysate was used to determine the protein concentration of each group of samples by using the BCA method. The activity was normalized to cellular protein and expressed as $\mathrm{U} / \mathrm{g}$.

Alizarin red staining and quantitative analysis. hBMMSCs were seeded into 12 -well plates at a density of $4 \times 10^{4}$ cells/well and cultured in osteogenic induction medium for 3 weeks before $1 \mathrm{ml}$ of $4 \%$ formaldehyde was added to each well at room temperature. After $30 \mathrm{~min}$, the formaldehyde solution was removed, and cells were washed twice with PBS. Cells were then treated with $0.5 \mathrm{ml}$ Alizarin red solution [Cyagen Biosciences (Guangzhou) Inc.] for $5 \mathrm{~min}$ at room temperature; following staining with Alizarin red dye, the cells were washed with PBS three times. Cells were analyzed under an optical microscope (Nikon Corporation, Tokyo, Japan; magnification, x100) to observe staining. After washing with PBS, 10\% cetylpyridinium chloride (Sigma-Aldrich; Merck KGaA) was added, and cells were lysed for $1 \mathrm{~h}$ at room temperature. The lysate was transferred to a 96-well plate, and the absorbance was measured at $570 \mathrm{~nm}$ using a 96-well-plate reader (Thermo Fisher Scientific, Inc.).

Flowcytometric analysisofapoptosis.hBMMSCs werecultured in 6-well plates at a density of $4 \times 10^{5}$ cells/well. Apoptosis was determined by flow cytometry using an Annexin V-fluorescein 


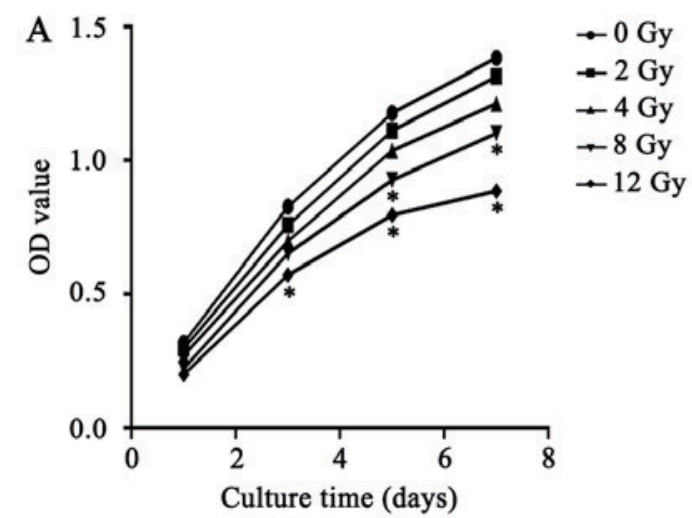

B
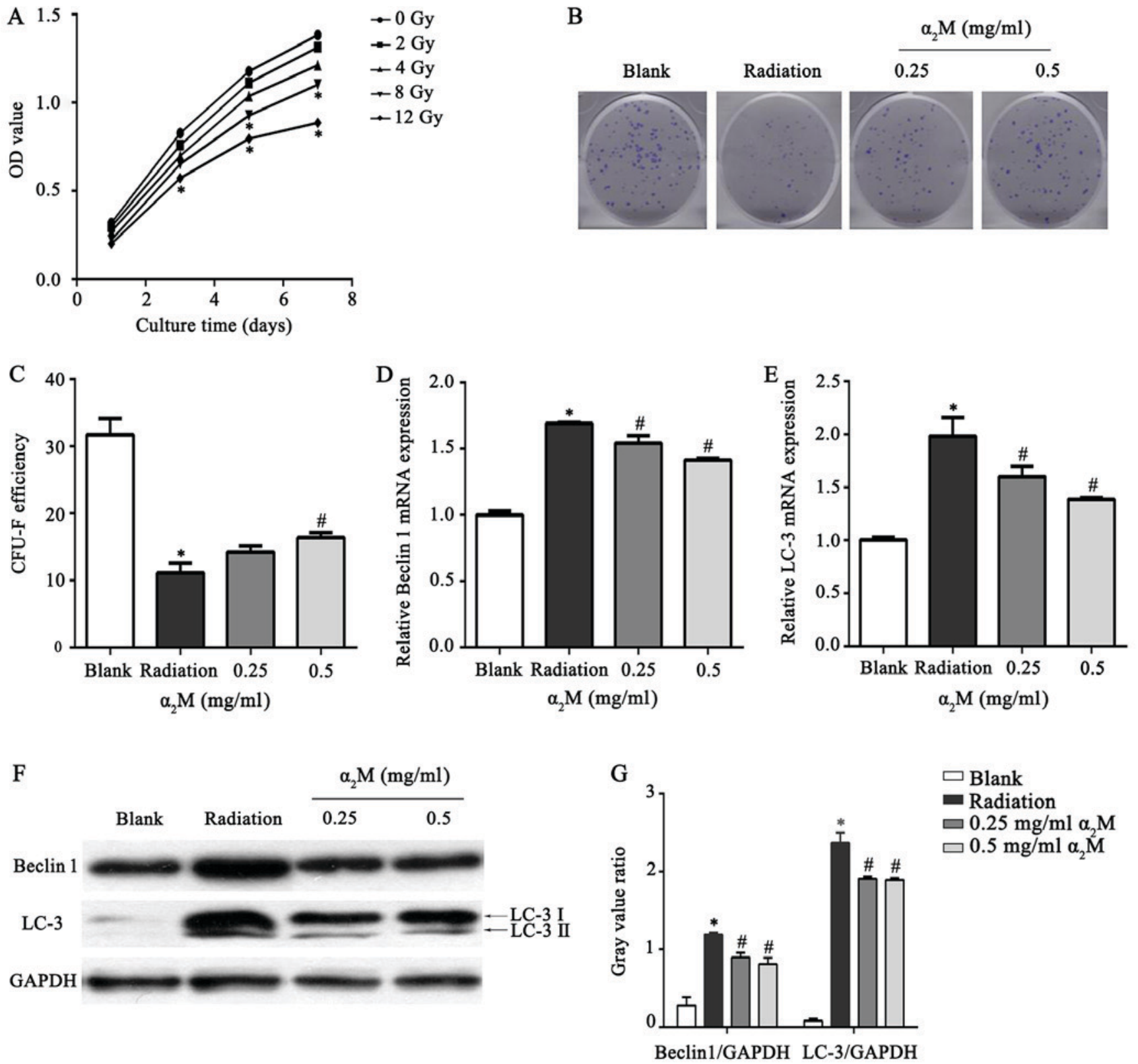

Figure 1. Effects of X-ray irradiation on hBMMSC proliferation and $\alpha_{2} \mathrm{M}$ on the CFU-F and expression of autophagy-associated genes in irradiated hBMMSCs. (A) Cell Counting Kit-8 was used to detect the effects of various doses of X-ray radiation on the proliferation of hBMMSCs on days 1,3,5 and 7 following irradiation. "P<0.05 vs. 0 Gy group ( $\mathrm{n}=3$ ). (B and C) A colony formation assay was performed to detect the effect of $\alpha_{2} \mathrm{M}$ on the CFU-F of hBMMSCs following radiation at $24 \mathrm{~h}$ (magnification, $\mathrm{x} 100)$. ${ }^{*} \mathrm{P}<0.05$ vs. blank control group, ${ }^{,} \mathrm{P}<0.05$ vs. radiation control group $(\mathrm{n}=3$ ). Reverse transcription-quantitative polymerase chain reaction was used to detect the effects of $\alpha_{2} \mathrm{M}$ on (D) Beclin1 and (E) LC-3 mRNA expression levels. (F and G) Western blotting was used to detect the effects of $\alpha_{2} \mathrm{M}$ on protein expression levels of Beclin1 and LC-3 in hBMMSCs at $24 \mathrm{~h}$ following irradiation. ${ }^{*} \mathrm{P}<0.05$ vs. blank control group, ${ }^{*} \mathrm{P}<0.05$ vs. radiation control group ( $\mathrm{n}=3$ ). $\alpha_{2} \mathrm{M}, \alpha$-2-macroglobulin; CFU-F, colony formation rate; hBMMSC, human bone marrow mesenchymal stem cells; LC-3, microtubule-associated protein $1 \mathrm{~A} / 1 \mathrm{~B} ; \mathrm{OD}$, optical density.

isothiocyanate (FITC)/propidium iodide (PI) staining kit (Nanjing KeyGen Biotech Co., Ltd., Nanjing, China). Cells were collected and stained in working solution (500 $\mu$ l binding buffer with $5 \mu \mathrm{l}$ Annexin V-FITC and $5 \mu \mathrm{l} \mathrm{PI}$ ) for $15 \mathrm{~min}$ at room temperature in the dark. Apoptotic cells were analyzed using a BD FACS Canto II flow cytometer running BD FACSDiva software version 4.1 (Franklin Lakes, NJ, USA). Annexin V-FITC single positive and Annexin V-FITC/PI double positive cells were considered apoptotic cells. The ratio of apoptotic cells to total cells was used to calculate the cell apoptosis rate.
SOD activity assay. hBMMSCs were incubated in 12-well plates at $4 \times 10^{4}$ cells/well, and $200 \mu 1$ RIPA lysis buffer was added to each well. Following lysis, $40 \mu 1$ lysate was obtained for analysis via a Superoxide Dismutase Assay kit (Nanjing Jiancheng Bioengineering Institute) according to the manufacturer's protocols. The absorbance values were measured at a wavelength of $450 \mathrm{~nm}$ using a 96-well-plate reader (Thermo Fisher Scientific, Inc.). The BCA method was applied using $10 \mu \mathrm{l}$ lysate to determine the protein concentration for each group of samples, in which the protein per milligram of SOD activity was determined. 
A

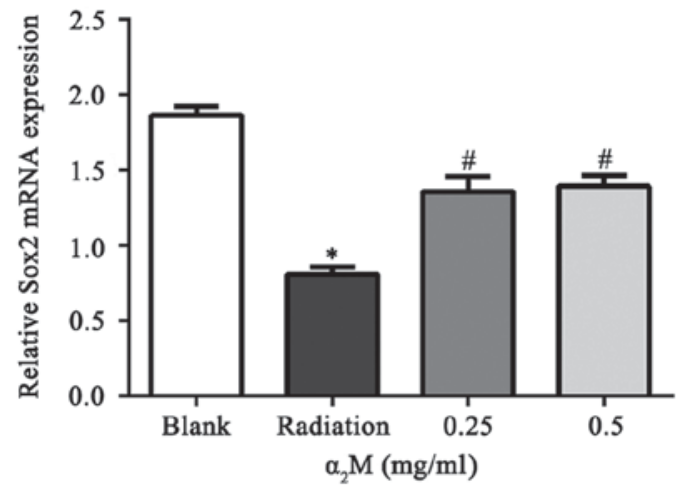

C

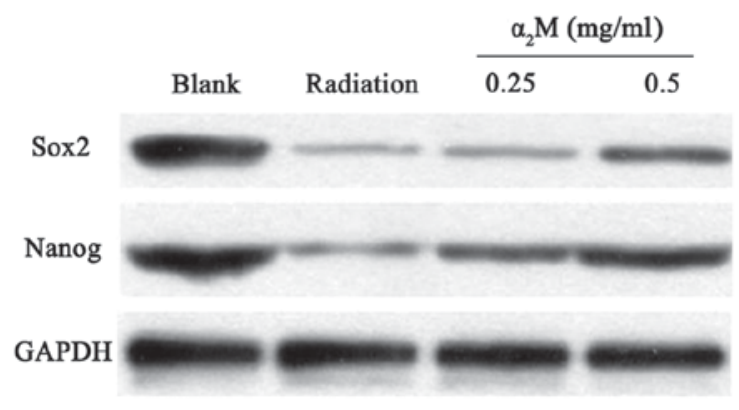

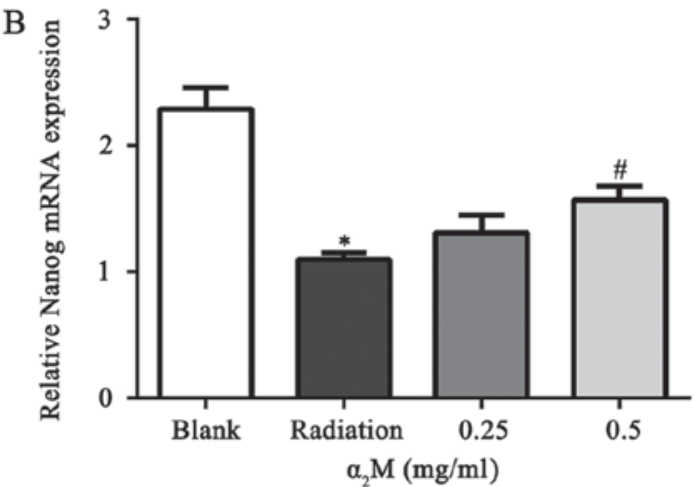

D

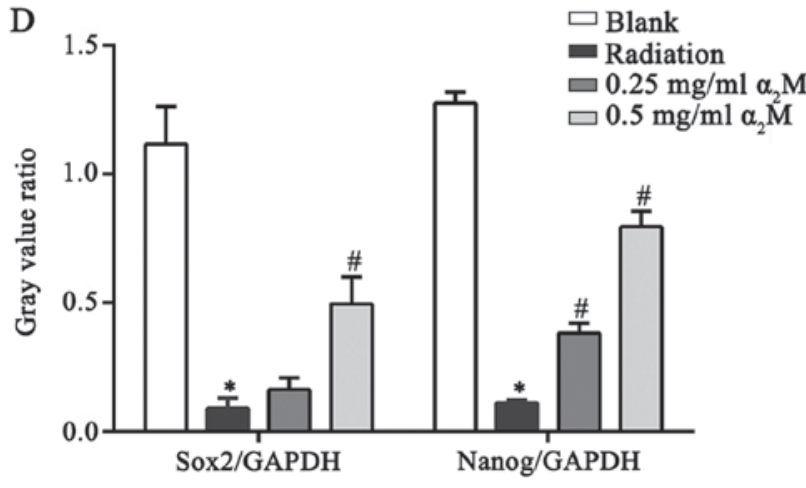

Figure 2. Effects of $\alpha_{2} \mathrm{M}$ on the expression of pluripotency-associated genes in irradiated hBMMSCs. Reverse transcription-quantitative polymerase chain reaction was employed to detect the effects of $\alpha_{2} \mathrm{M}$ on the mRNA expression levels of (A) Sox2 and (B) Nanog in hBMMSCs at 24 h following radiation. (C and D) Western blotting was employed to detect the effects of $\alpha_{2} \mathrm{M}$ on the protein expression levels of Sox 2 and Nanog in hBMMSCs at $24 \mathrm{~h}$ following radiation. ${ }^{*} \mathrm{P}<0.05$ vs. blank control group, ${ }^{\#} \mathrm{P}<0.05$ vs. radiation control group $(\mathrm{n}=3)$. hBMMSC, human bone marrow mesenchymal stem cells; $\alpha_{2} \mathrm{M}$, $\alpha$-2-macroglobulin; Sox 2, sex determining region Y.

Detection of reactive oxygen species (ROS) levels. hBMMSCs were cultured in 6 -well plates at $4 \times 10^{5}$ cells/well in complete medium. 2',7'-Dichlorodihydrofluorescein diacetate (Nanjing Jiancheng Bioengineering Institute) was diluted 1:1,000 with serum-free cell culture medium and incubated in the cell incubator for $20 \mathrm{~min}$ at $37^{\circ} \mathrm{C}$. The cells were washed three times in serum-free cell culture medium, and analyzed under a Leica TCS-SP8 confocal microscope (Leica Microsystems GmbH, Wetzlar, Germany; magnification, x100). An excitation wavelength of $502 \mathrm{~nm}$ was applied and images of the cells were collected.

Statistical analysis. All experiments were performed at least three times. The results were analyzed using SPSS software, version 20.0 (IBM Corp., Armonk, NY, USA) and are expressed as the mean \pm standard deviation. The data were analyzed by one-way analysis of variance, followed by a Bonferroni post-hoc test for multiple comparisons. $\mathrm{P}<0.05$ was considered to indicate a statistically significant difference.

\section{Results}

$X$-ray inhibits $h B M M S C$ proliferation, $\alpha_{2} M$ increases hBMMSC colony formation rate and reduces autophagy following radiation. hBMMSC proliferation decreased with increasing doses of radiation; the hBMMSCs treated with 8 and 12 Gy radiation exhibited significantly lower proliferation rates than the 0 Gy-treated group. Cell proliferation was too low in the 12 Gy group after 5 and 7 days of treatment; thus, 8 Gy was selected for follow-up experiments (Fig. 1A). The colony formation experiment demonstrated that hBMMSCs irradiated with 8 Gy X-rays and cultured with $0.50 \mathrm{mg} / \mathrm{ml}$ $\alpha_{2} \mathrm{M}$ exhibited a significantly increased colony formation rate compared with in the irradiated group; however, the colony formation rate of the irradiated group was significantly decreased compared with in the control (Fig. 1B and C). RT-qPCR revealed that $\alpha_{2} \mathrm{M}$ significantly downregulated the mRNA expression levels of Beclin1 and LC-3 in hBMMSCs in a dose-dependent manner compared with the irradiation group. Treatment with $0.50 \mathrm{mg} / \mathrm{ml} \alpha_{2} \mathrm{M}$ appeared to markedly downregulate the mRNA expression levels of Beclin and LC-3 compared with the $0.25 \mathrm{mg} / \mathrm{ml} \alpha_{2} \mathrm{M}$-treated group; however, no notable differences were observed in the protein expression levels of LC-3 between the two $\alpha_{2} \mathrm{M}$ treatment groups (Fig. 1D-G).

$\alpha_{2} M$ increases the pluripotency of $h B M M S C s$ following irradiation. RT-qPCR revealed that the mRNA expression levels of Sox 2 and Nanog were significantly upregulated at doses of $0.50 \mathrm{mg} / \mathrm{ml} \alpha_{2} \mathrm{M}$, compared with in the radiation group; however, at doses of $0.25 \mathrm{mg} / \mathrm{ml} \alpha_{2} \mathrm{M}$, Nanog mRNA expression was only markedly upregulated whereas Sox2 was significantly upregulated. Protein expression levels of Nanog and Sox 2 were also significantly upregulated at doses of $0.50 \mathrm{mg} / \mathrm{ml} \alpha_{2} \mathrm{M}$, compared with in the radiation group however, at doses of $0.25 \mathrm{mg} / \mathrm{ml} \alpha_{2} \mathrm{M}$, Sox 2 protein levels were markedly upregulated (Fig. 2A-D). 

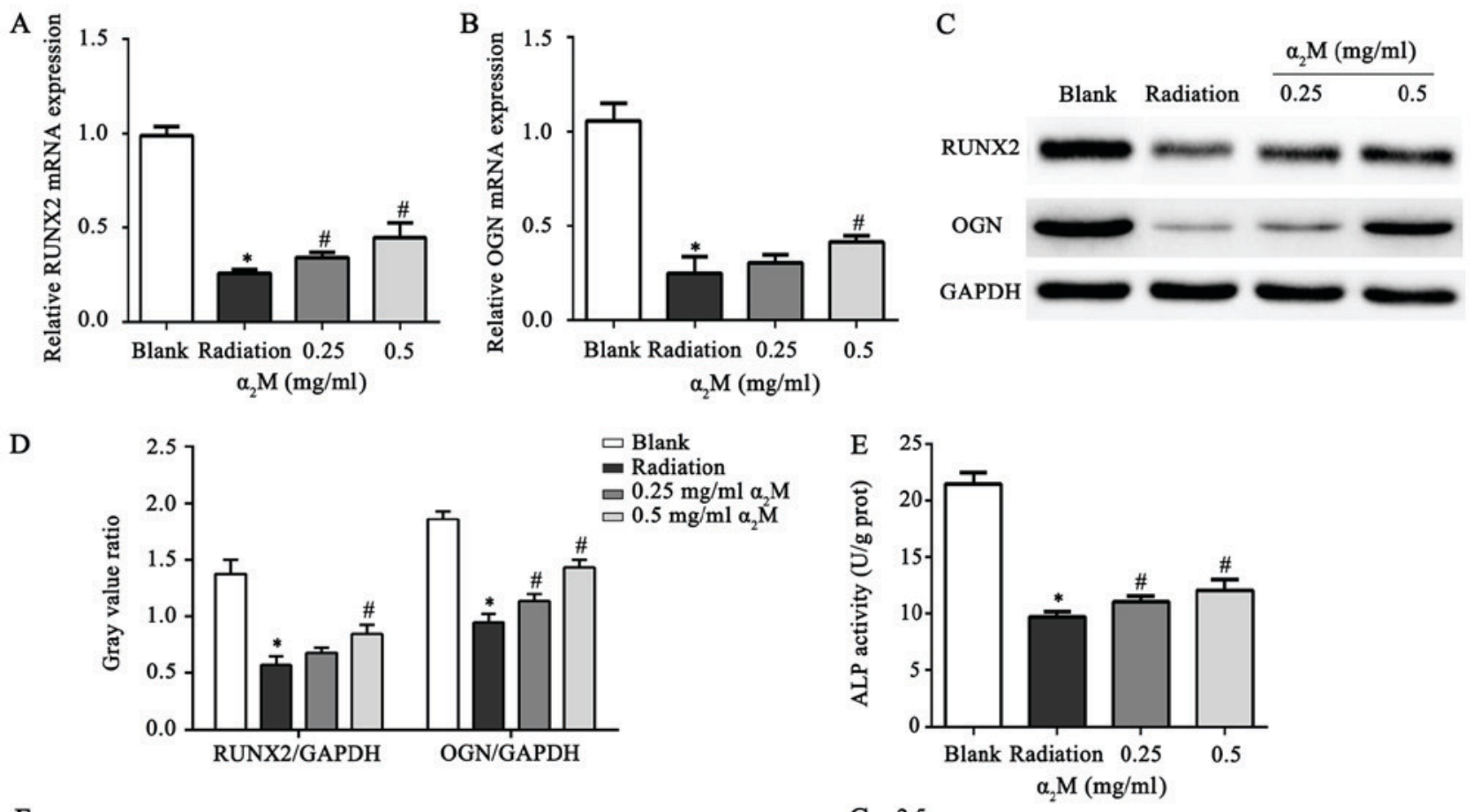

F
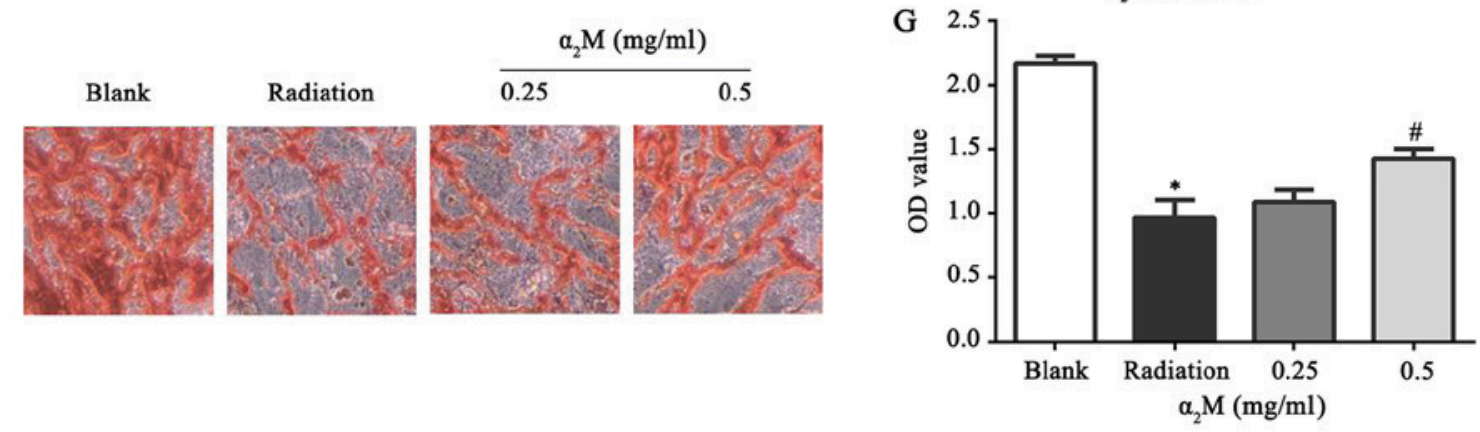

Figure 3. Effects of $\alpha_{2} \mathrm{M}$ on osteogenic differentiation potential of irradiated hBMMSCs. Reverse transcription-quantitative polymerase chain reaction was used to detect the effect of $\alpha_{2} \mathrm{M}$ on the mRNA expression of (A) RUNX2 after 1 week and (B) OGN after 2 weeks of osteogenesis induction in irradiated hBMMSCs. " $\mathrm{P}<0.05$ vs. blank control group, ${ }^{\#} \mathrm{P}<0.05$ vs. radiation control group ( $\mathrm{n}=3$ ). (C and $\left.\mathrm{D}\right)$ Western blotting was used to detect the effects of $\alpha_{2} \mathrm{M}$ on the protein expression levels of RUNX2 after 1 week and OGN after 2 weeks of osteogenesis induction in irradiated hBMMSCs. ${ }^{*} \mathrm{P}<0.05$ vs. blank control group, ${ }^{\#} \mathrm{P}<0.05$ vs. radiation control group $\left(\mathrm{n}=3\right.$ ). (E) Effects of $\alpha_{2} \mathrm{M}$ on ALP activity after 1 week of osteogenesis induction in irradiated hBMMSCs. ${ }^{*} \mathrm{P}<0.05$ vs. blank control group, ${ }^{\sharp} \mathrm{P}<0.05$ vs. radiation control group $(\mathrm{n}=3)$. ( $\mathrm{F}$ and $\left.\mathrm{G}\right)$ Alizarin red staining was used to detect the effects of $\alpha_{2} \mathrm{M}$ on the formation of calcium nodules after 3 weeks of osteogenesis induction in irradiated hBMMSCs (magnification, $\mathrm{x} 100$ ). ${ }^{*} \mathrm{P}<0.05$ vs. blank control group, ${ }^{*} \mathrm{P}<0.05$ vs. radiation control group $(n=3) . \alpha_{2} \mathrm{M}, \alpha$-2-macroglobulin; ALP, alkaline phosphatase; hBMMSC, human bone marrow mesenchymal stem cells; OD, optical density; OGN, osteoglycin; RUNX2, runt-related transcription factor 2.

$\alpha_{2} M$ increases the osteogenic differentiation ability of hBMMSCs following irradiation. The mRNA and protein expression levels of RUNX2 and OGN were significantly increased with the addition of $\alpha 2 \mathrm{M}$ following X-ray irradiation compared with in the radiation group; however, treatment with $0.25 \mathrm{mg} / \mathrm{ml}$ only markedly increased the protein expression level of RUNX2 (Fig. 3A-D). In addition, ALP activity significantly increased in response to $\alpha_{2} \mathrm{M}$ treatment compared with in the radiation group (Fig. 3E). Microscopic analysis revealed the formation of calcium nodules and the degree of staining increased (Fig. 3F and G).

$\alpha_{2} M$ reduces the rate of apoptosis and improves the antioxidative abilities of $h B M M S C s$ following irradiation. hBMMSCs were irradiated with X-rays and then treated with $\alpha_{2} \mathrm{M}$. The rate of apoptosis was significantly decreased in response to treatment with $0.50 \mathrm{mg} / \mathrm{ml} \alpha_{2} \mathrm{M}$ compared with in the irradiated group (Fig. 4A and B). Additionally, treatment with
$0.50 \mathrm{mg} / \mathrm{ml} \alpha_{2} \mathrm{M}$ significantly increased SOD activity and the protein expression levels of MnSOD compared with in the radiation group (Fig. 4C-E). The levels of ROS were notably decreased following treatment with $\alpha_{2} \mathrm{M}$ compared with in the radiation group (Fig. 4F).

\section{Discussion}

ORNJ, a serious complication of head and neck radiotherapy, often results in poor prognosis. Investigations into protective strategies against radiation are required to minimize radiotherapy-induced damage to normal tissue. The development of efficient, low-toxicity radiation protectants to reduce and prevent the occurrence of symptoms associated with radiation sickness, such as ORNJ, is an important area of radiation protection research. Certain drugs can serve anti-radiation roles; however, due to toxic side effects, their application is not recommended for the treatment of malignancies. 

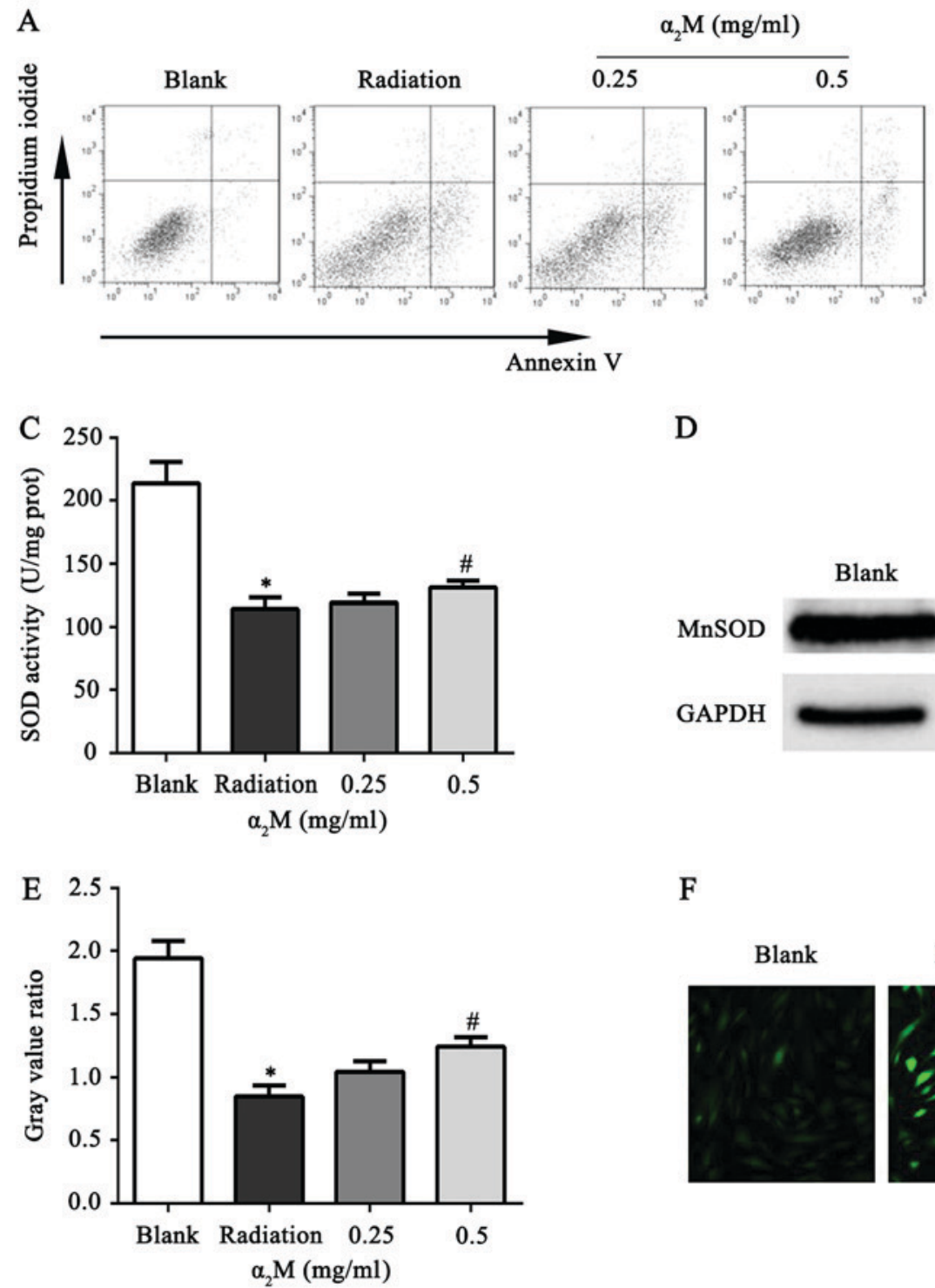

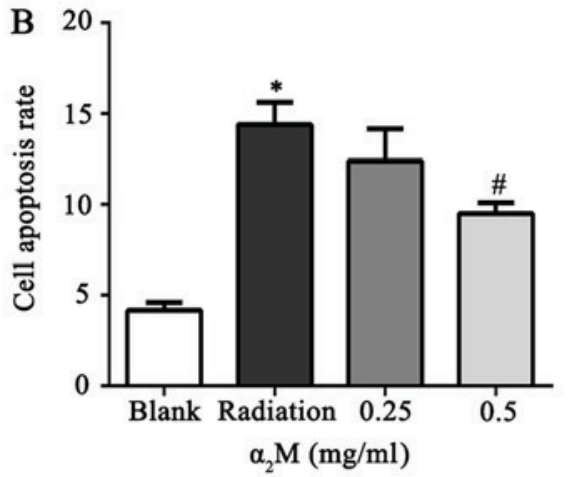

D

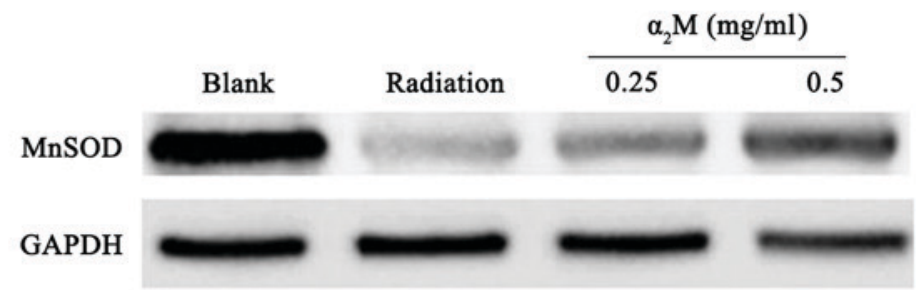

F Radiation

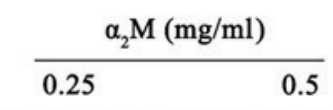
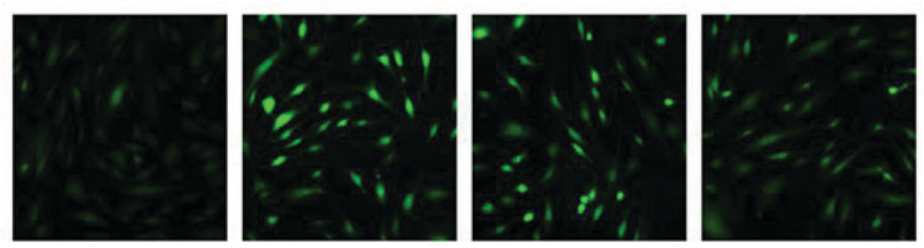

Figure 4. Effects of $\alpha_{2} \mathrm{M}$ on the rate of apoptosis and the antioxidative capacity of irradiated hBMMSCs. (A and B) Effects of $\alpha_{2} \mathrm{M}$ on the rate of apoptosis in hBMMSCs at $24 \mathrm{~h}$ following radiation. ${ }^{*} \mathrm{P}<0.05$ vs. blank control group, ${ }^{"} \mathrm{P}<0.05$ vs. radiation control group $(\mathrm{n}=3)$. (C) Effects of $\alpha_{2} \mathrm{M}$ on SOD activity in hBMMSCs at $24 \mathrm{~h}$ following radiation. "P $<0.05$ vs. blank control group, ${ }^{*} \mathrm{P}<0.05$ vs. radiation control group ( $\mathrm{n}=3$ ). (D and $\left.\mathrm{E}\right)$ Western blotting was used to detect the effects of $\alpha_{2} \mathrm{M}$ on the protein expression levels of MnSOD in hBMMSCs at $24 \mathrm{~h}$ following radiation. ${ }^{*} \mathrm{P}<0.05$ vs. blank control group, ${ }^{~} \mathrm{P}<0.05$ vs. radiation control group $(\mathrm{n}=3)$. (F) Effects of $\alpha_{2} \mathrm{M}$ on the levels of ROS in hBMMSCs at $24 \mathrm{~h}$ following radiation. $\alpha_{2} \mathrm{M}, \alpha$-2-macroglobulin; hBMMSC, human bone marrow mesenchymal stem cells; MnSOD, manganese superoxide dismutase; ROS, reactive oxygen species.

The pathogenesis of ORNJ is not completely understood; however, in the majority of cases, the balance of bone reshaping is disrupted, and is accompanied by the accumulation of extracellular matrix and microvascular thrombosis, ultimately leading to necrosis of bone tissue (14). BMMSCs are a cell type with pluripotent differentiation potential and are one of the cell types most affected by bone tissue radiation injury. Studies have reported that the renewal and formation of bone tissue is a continuous process, which mainly involves the differentiation of BMMSCs into osteogenic progenitor cells that gradually mature into differentiated osteoblasts. In the process of bone remodeling, dysregulation of the balance between bone formation and bone resorption balance may lead to bone defects (15).

In a previous study, the authors reported that the serum from patients with ORNJ contains 12 proteins, which exhibits differential expression, including $\alpha_{2} \mathrm{M}$ and three additional proteins with abnormally low expression levels. By expanding the clinical sample size, it was demonstrated that $\alpha_{2} \mathrm{M}$ expression was low in the serum of patients with ORNJ, and patients with nasopharyngeal carcinoma exhibited a positive correlation between serum $\alpha_{2} \mathrm{M}$ levels and radiotherapy dose. These findings suggested that serum $\alpha_{2} \mathrm{M}$ and radiation damage from jaw ionization may be closely associated; however, the underlying mechanism requires further investigation.

$\alpha_{2} \mathrm{M}$ is a member of an important class of macromolecules in the plasma, which contains a broad spectrum of protease inhibitors. In recent years, accumulating evidence has revealed that $\alpha_{2} \mathrm{M}$ has a wide range of biological functions, including antioxidant activity against ROS and anti-ionizing radiation effects. It was reported that $\alpha_{2} \mathrm{M}$ can remove oxygen free radicals by increasing the activity of $\mathrm{Mn} /$ copper SOD and reduce 
the extent of DNA damage, which may induce protection against radiation in liver tissue (16). This finding suggested that $\alpha_{2} \mathrm{M}$ may possess an important protective effect against tissue damage caused by ionizing radiation.

In the present study, hBMMSCs were subjected to high doses of X-ray irradiation and treated with 0.25 and $0.50 \mathrm{mg} / \mathrm{ml}$ of $\alpha_{2} \mathrm{M}$ to determine whether $\alpha_{2} \mathrm{M}$ exerts protective effects against radiation damage. High doses of X-rays can induce the abnormal proliferation of normal hBMMSCs and cause DNA damage. The present study reported that the proliferative ability of hBMMSCs treated with X-ray irradiation was inhibited in a dose-dependent manner. The proliferative ability of hBMMSCs irradiated at 12 Gy was notably low. Therefore, 8 Gy was selected as the experimental dose in the present study.

The colony formation rate is the rate of cell survival, which indicates the number of cloned cells that have survived and divided following cell inoculation. This reflects two important traits: Cell population dependence and proliferative ability (17). Autophagy is a conserved regulatory mechanism of cells that functions via structural remodeling within the cell, material renewal processes, and adaptive responses of cells to injury stimuli $(18,19)$. Beclin1 and LC-3 are important genes involved in the initiation of autophagy (20); the expression levels of these two genes may partly reflect the extent of autophagy of cells (20). In the present study, it was observed that $\alpha_{2} \mathrm{M}$ increased the colony formation rate of hBMMSCs following irradiation and downregulated the gene expression levels of Beclin1 and LC-3, thereby reducing cell autophagy. These results suggest that $\alpha_{2} \mathrm{M}$ may protect hBMMSCs by inducing the autophagy protective mechanism and increasing the cell proliferation rate.

Sox 2 and Nanog are cytokines which are employed as markers of stem cell pluripotency and serve key roles in maintaining stem cell function $(21,22)$. Dysregulation in the expression of these cytokines are associated with alterations in the pluripotent differentiation characteristics of stem cells. In addition, the present study investigated the effects of $\alpha_{2} \mathrm{M}$ on the osteogenic differentiation potential of irradiated hBMMSCs. ALP is one of the enzymes necessary for cell ossification and is an early marker of osteoblasts; ALP expression increases during osteogenic differentiation $(23,24)$. RUNX2 is a well-reported characteristic marker gene associated with the early stages of bone formation and is an important transcription factor for the early stage of osteogenic differentiation $(25,26)$. OGN is a secretory protein in bone tissue, which serves an important role in inhibiting osteoclasts and inducing osteogenesis. OGN has been reported as an osteoinductive factor that can promote bone formation following binding with transforming growth factor- $\beta 1$ or $-\beta 2$ (27). Calcium nodules represent the final stage of osteogenic differentiation and are produced by the mineralization of osteoblasts (28).

The results of the present study demonstrated that $\alpha_{2} \mathrm{M}$ increased the expression levels of Sox 2 and Nanog in hBMMSCs following irradiation. The effects $0.50 \mathrm{mg} / \mathrm{ml} \alpha_{2} \mathrm{M}$ were further increased compared with $0.25 \mathrm{mg} / \mathrm{ml} \alpha_{2} \mathrm{M}$ and indicated that $\alpha_{2} \mathrm{M}$ may alleviate multipotent differentiation injury induced by X-rays. In addition, $\alpha_{2} \mathrm{M}$ at 0.25 or $0.50 \mathrm{mg} / \mathrm{ml}$ was added to the osteogenic induction differentiation medium for 3 days following irradiation. Compared with in the untreated control group, the treated groups exhibited increased ALP activities and upregulated gene expression levels of RUNX2 and OGN; a further increase in effects was observed in the group treated with $0.50 \mathrm{mg} / \mathrm{ml} \alpha_{2} \mathrm{M}$ compared with $0.25 \mathrm{mg} / \mathrm{ml} \alpha_{2} \mathrm{M}$. In addition, increased calcium nodule formation was observed in accordance with an increased degree of staining. This indicated that $\alpha_{2} \mathrm{M}$ may alleviate radiation damage to hBMMSCs and improve their osteogenic differentiation ability, further verifying the protective effects of $\alpha_{2} \mathrm{M}$ on the osteogenic differentiation of hBMMSCs.

Tissue damage caused by ionizing radiation arises not only from the direct damage of macromolecules but also from the formation of superoxide anion molecules, such as ROS. These molecules possess oxidative strong abilities, and are capable of inducing the degeneration of macromolecules such as DNA, via oxidation. This results in damage to cell structure and function, eventually leading to tissue damage and genetic mutations. ROS are classic indicators for the degree of oxidative stress $(29,30)$. In addition, ROS can initiate chain reactions, and react with a variety of unsaturated fatty acids and cholesterol on the cell membrane, resulting in oxidative damage to cells and eventually apoptosis. Cell death via apoptosis serves a role in maintaining normal tissue morphology and cell death, which are under the control of apoptosis-associated genes $(31,32)$.

Using immunofluorescence, Hou et al (33) reported that the irradiation of hBMMSCs with 6 Gy of X-rays results in increased intracellular ROS levels, further confirming that ionizing radiation increases the levels of ROS, resulting in DNA damage, decreasing the ability of hBMMSCs to differentiate into multiple cell types. SOD is an important factor associated with the removal of free radicals in the body by antioxidant enzymes, and is known as the first line of antioxidative defense of the body.

Antioxidative removal of ROS is an important mechanism for improving the activity of SOD (34). In the present study, hBMMSCs were treated with 8 Gy X-ray radiation and then treated with $\alpha_{2} \mathrm{M}$. After $24 \mathrm{~h}$, the activity of SOD and expression levels of MnSOD protein were significantly increased, the level of ROS decreased, and the rate of apoptosis decreased. This suggested that $\alpha_{2} \mathrm{M}$ may reduce the oxidative stress response induced by the irradiation of hBMMSCs and thus serve a role in prevention of radiation injury.

In conclusion, the present study proposed the possibility of using $\alpha_{2} \mathrm{M}$ to protect against bone tissue radiation injury. Further investigation with systematic experiments is required to determine the specific mechanism of action underlying $\alpha_{2} \mathrm{M}$ in the prevention and treatment of ORNJ in the future.

\section{Acknowledgements}

The authors thank Professor Huanliang Liu (Guangdong Provincial Key Laboratory of Colorectal and Pelvic Floor Diseases, Guangdong Institute of Gastroenterology and the Sixth Affiliated Hospital, Sun Yat-sen University, Guangzhou, China) for providing technical assistance and Professor Yi Liu (Laboratory of Tissue Regeneration and Immunology and Department of Periodontics, Beijing Key Laboratory of Tooth Regeneration and Function Reconstruction, School of 
Stomatology, Capital Medical University, Beijing, China) for guidance during revision of the manuscript.

\section{Funding}

The present study was supported by the Natural Science Foundation of Guangdong Province (grant no.2015A030313064), and the Science and Technology Planning Project of Guangdong Province (grant nos. 2014A020212127 and 2017A010105027).

\section{Availability of data and materials}

All data generated or analyzed during this study are included in this published article.

\section{Authors' contributions}

YL, WC, XK, JL and SF made substantial contributions to the concept and design of the present study. YL, WC, JL and XC cultured human bone marrow mesenchymal stem cells. YL, WC and YG performed the experiments. YL, WC, WZ and SF acquired and analyzed the data. YL, WC, XC and SF wrote the manuscript. All the authors read and approved the final version of the manuscript.

\section{Ethics approval and consent to participate}

Not applicable.

\section{Patient consent for publication}

Not applicable.

\section{Competing interests}

The authors declare that they have no competing interests.

\section{References}

1. Bots WTC, van den Bosch S, Zwijnenburg EM, Dijkema T, van den Broek GB, Weijs WLJ, Verhoef LCG and Kaanders JHAM: Reirradiation of head and neck cancer: Long-term disease control and toxicity. Head Neck 39: 1122-1130, 2017.

2. Nadella KR, Kodali RM, Guttikonda LK and Jonnalagadda A: Osteoradionecrosis of the Jaws: Clinico-therapeutic management: A literature review and update. J Maxillofac Oral Surg 14 891-901, 2015.

3. Dhanda J, Pasquier D, Newman L and Shaw R: Current concepts in osteoradionecrosis after head and neck radiotherapy. Clin Oncol (R Coll Radiol) 28: 459-466, 2016.

4. Moroni L and Fornasari PM: Human mesenchymal stem cells: A bank perspective on the isolation, characterization and potential of alternative sources for the regeneration of musculoskeletal tissues. J Cell Physiol 228: 680-687, 2013.

5. Baker N, Boyette LB and Tuan RS: Characterization of bone marrow-derived mesenchymal stem cells in aging. Bone 70 : 37-47, 2015.

6. Wang Y, Zhu G, Wang J and Chen J: Irradiation alters the differentiation potential of bone marrow mesenchymal stem cells. Mol Med Rep 13: 213-223, 2016.

7. Fang S, Chen W, Wang S, Kong X, Sun X, Ge Y and Hu K: Proteomic Analysis of Serum in patients with Osteoradionecrosis of the Jaws. Int Dental J 62: 24, 2012.

8. Fang S, Kong X, Chen W, Chen Y, He F, Ge Y, Sun X and Wang S: Identification of differential proteins that related to bone metabolism in the serum of ORNJ patients. Int Dental J 62: 25 , 2012.
9. Chen X, Kong X, Cao W, Ge Y, Wang S, Zhang Z, Li H, Liu Y and Fang S: The detection of serum and urine levels of alpha 2-Macroglobulin in nasopharyngeal carcinoma patients receiving late period radiotherapy and its significance. Int J Dentistry Oral Sci 5: 611-617, 2018.

10. Rehman AA, Ahsan H and Khan FH: a2-macroglobulin: A physiological guardian. J Cell Physiol 228: 1665-1675, 2013.

11. Rehman AA, Zaman M, Zia MK, Ahsan H, Khan RH and Khan FH: Conformational behavior of alpha-2-macroglobulin: Aggregation and inhibition induced by TFE. Int J Biol Macromol 104: 539-546, 2017.

12. Chen X, Kong X, Zhang Z, Chen W, Chen J, Li H, Cao W, Ge Y and Fang S: Alpha-2-macroglobulin as a radioprotective agent: A review. Chin J Cancer Res 26: 611-621, 2014.

13. Livak KJ and Schmittgen TD: Analysis of relative gene expression data using real-time quantitative PCR and the 2(-Delta Delta C(T)) method. Methods 25: 402-408, 2001.

14. Lyons A and Ghazali N: Osteoradionecrosis of the jaws: Current understanding of its pathophysiology and treatment. Br J Oral Maxillofac Surg 46: 653-660, 2008.

15. Wissing MD: Chemotherapy- and irradiation-induced bone loss in adults with solid tumors. Curr Osteoporos Rep 13: 140-145, 2015.

16. Bogojević D, Poznanović G, Grdović N, Grigorov I, Vidaković M, Dinić S and Mihailović M: Administration of rat acute-phase protein $\alpha(2)$-macroglobulin before total-body irradiation initiates cytoprotective mechanisms in the liver. Radiat Environ Biophys 50: 167-179, 2011.

17. Aizman I, Holland WS, Yang C and Bates D: $\alpha$ SMA expression in large colonies of Colony-forming units-fibroblast as an early predictor of bone marrow MSC expandability. Cell Med 8: 79-85, 2016.

18. Liang DH, El-Zein R and Dave B: Autophagy Inhibition to Increase Radiosensiti-zation in breast cancer. J Nucl Med Radiat Ther 6: 254, 2015.

19. Koukourakis MI, Mitrakas AG and Giatromanolaki A: Therapeutic interactions of autophagy with radiation and temozolomide in glioblastoma: Evidence and issues to resolve. $\mathrm{Br}$ J Cancer 114: 485-496, 2016.

20. Kim M, Fekadu J, Maronde E and Rami A: Alleviation of autophagy by knockdown of Beclin-1 enhances susceptibility of hippocampal neurons to proapoptotic signals indeced by amino acid starvation. Histochem Cell Biol 139: 99-108, 2013.

21. Go MJ, Takenaka C and Ohgushi H: Forced expression of Sox2 or Nanog in human bone marrow derived mesenchymal stem cells maintains their expansion and differentiation capabilities. Exp Cell Res 314: 1147-1154, 2008.

22. Heffernan C, Sumer H and Verma PJ: Generation of clinically relevant 'induced pluripotent stem' (iPS) cells. J Stem Cells 6: 109-127, 2011.

23. Millán JL: The role of phosphatases in the initiation of skeletal mineralization. Calcif Tissue Int 93: 299-306, 2013.

24. Halling Linder C, Enander K and Magnusson P: Glycation contributes to interaction between human bone alkaline phosphatase and collagen type I. Calcif Tissue Int 98: 284-293, 2016.

25. Henriquez B, Hepp M, Merino P, Sepulveda H, van Wijnen AJ, Lian JB, Stein GS, Stein JL and Montecino M: C/EBP $\beta$ binds the P1 promoter of the Runx2 gene and up-regulates Runx2 transcription in osteoblastic cells. J Cell Physiol 226: 3043-3052, 2011.

26. Qin X, Jiang Q, Matsuo Y, Kawane T, Komori H, Moriishi T, Taniuchi I, Ito K, Kawai Y, Rokutanda S, et al: Cbfb regulates bone development by stabilizing runx family proteins. J Bone Miner Res 30: 706-714, 2015

27. Tanaka K, Matsumoto E, Higashimaki Y, Katagiri T, Sugimoto T, Seino S and Kaji H: Role of osteoglycin in the linkage between muscle and bone. J Biol Chem 287: 11616-11628, 2012.

28. Gregory CA, Gunn WG, Peister A and Prockop DJ: An Alizarin red-based assay of mineralization by adherent cells in culture: Comparison with cetylpyridinium chloride extraction. Anal Biochem 329: 77-84, 2004.

29. Sakuma S, Abe M, Kohda T and Fujimoto Y: Hydrogen peroxide generated by xanthine/xanthine oxidase system represses the proliferation of colorectal cancer cell line Caco-2. J Clin Biochem Nutr 56: 15-19, 2015.

30. Ivanov VE, Usacheva AM, Chernikov AV, Bruskov VI and Gudkov SV: Formation of long-lived reactive species of blood serum proteins induced by low-intensity irradiation of helium-neon laser and their involvement in the generation of reactive oxygen species. J Photochem Photobiol B 176: 36-43, 2017. 
31. Wu J, Liu S, Meng H, Qu T, Fu S, Wang Z, Yang J, Jin D and Yu B: Neuropeptide $Y$ enhances proliferation and prevents apoptosis in rat bone marrow stromal cells in association with activation of the Wnt $/ \beta$-catenin pathway in vitro. Stem Cell Res 21: 74-84, 2017.

32. Deng B, Jiang H, Zeng K, Liang Y, Wu Y and Yang Y: Removal from adherent culture contributes to apoptosis in human bone marrow mesenchymal stem cells. Mol Med Rep 15: 3499-3506, 2017.

33. Hou J, Han ZP, Jing YY, Yang X, Zhang SS, Sun K, Hao C, Meng Y, Yu FH, Liu XQ, et al: Autophagy prevents irradiation injury and maintains stemness through decreasing ROS generation in mesenchymal stem cells. Cell Death Dis 4: e844, 2013.
34. M Fetherolf M, Boyd SD, Winkler DD and Winge DR: Oxygen-dependent activation of $\mathrm{Cu}, \mathrm{Zn}$-superoxide dismutase-1. Metallomics 9: 1047-1059, 2017.

(i) () $($ This work is licensed under a Creative Commons EY No No Attribution-NonCommercial-NoDerivatives 4.0 International (CC BY-NC-ND 4.0) License. 